\title{
A MONOTONICITY THEOREM FOR THE FAMILY $f_{a}(x)=a-x^{2}$
}

\author{
LEO JONKER
}

\begin{abstract}
Let $f_{a}(x)=a-x^{2}, x \in\left[-\frac{1}{2}-\frac{1}{2} \sqrt{1+4 a}, \frac{1}{2}+\frac{1}{2} \sqrt{1+4 a}\right]$ and $a \in[0,2]$. It is proved that if $f_{a}$ has a periodic orbit of odd period $n$ and if $b>a$, then $f_{b}$ has a periodic orbit of period $n$. This is equivalent to the corresponding result for the function family $g_{\lambda}(x)=\lambda x(1-x), x \in[0,1]$, $\lambda \in[0,4]$.
\end{abstract}

Among one-dimensional discrete dynamical systems those which are simplest and best understood are the unimodal functions of an interval into itself. These functions can be analysed with the help of Milnor and Thurston's kneading theory $[7,3]$ which associates to the function $f$ a formal power series $\nu(f) \in \mathbf{Z}[[t]]$ called variously the kneading invariant or (in [7]) the kneading determinant. The kneading invariant almost completely specifies the periodic orbits and completely specifies the topological entropy of $f[\mathbf{7}, \mathbf{3}, \mathbf{4}, \mathbf{5}]$. If in addition the function $f$ has negative Schwarzian derivative, then the family of unimodal functions with the same kneading invariant either constitutes a conjugacy class of such functions or else is the union of two conjugacy classes [2]. Thus the kneading invariant almost characterizes $f$ up to conjugacy. Milnor and Thurston showed that in a $C^{1}$ family of unimodal functions $f_{a}$ the topological entropy is a continuous function of the parameter $a$ (see [7]). The bifurcations in such a family are completely understood, and occur in a versal pattern that is related to Sarkovskii's ordering of the natural numbers [6]. However, there is no function family $f_{a}$ for which it is known that $\nu\left(f_{a}\right)$ is a monotone function of $a$ or that the topological entropy $h\left(f_{a}\right)$ is monotone in the parameter. Milnor and Thurston conjecture that the family of functions

$$
g_{\lambda}(x)=\lambda x(1-x), \quad x \in[0,1],
$$

has this property. Indeed computer calculations seem to indicate that $h\left(g_{\lambda}\right)$ is monotone increasing.

In this paper we present a modest result in this direction. It is known that the topological entropy increases monotonically with the appearance of periodic orbits of new periods. We prove the following.

THEOREM. If $g_{\lambda}$ has a periodic orbit of odd period $n$ and if $\mu>\lambda$, then $g_{\mu}$ has a periodic orbit of period $n$.

Let

$$
f_{a}(x)=a-x^{2}
$$

Received by the editors August 28, 1981.

1980 Mathematics Subject Classification. Primary 58F20.

Key words and phrases. Mapping on an interval, periodic points, monotonicity.

(C) 1982 American Mathematical Society 0002-9939/81/0000-0730/\$02.00 
where $x \in I_{a}=\left[-\frac{1}{2}-\frac{1}{2} \sqrt{1+4 a}, \frac{1}{2}+\frac{1}{2} \sqrt{1+4 a}\right]$ and where $a \in[0,2]$. Since $\varsigma(x)=(x /(1+\sqrt{1+4 a}))+\frac{1}{2}$ is a conjugacy between $f_{a}$ and $g_{\lambda}$ with $\lambda=(1+$ $\sqrt{1+4 a})$, we prove the theorem for the function family $f_{a}$.

Both families are unimodal in the sense that $f^{\prime}(x)>0$ if $x<c$ and $f^{\prime}(x)<0$ if $x>c$ where $c$ is the unique critical point.

Note that

$$
\frac{\partial}{\partial a} f_{a}^{n}(x)=1-2 f_{a}^{n-1}(x) \cdot \frac{\partial}{\partial a} f_{a}^{n-1}(x)
$$

Suppose now that $x_{0}, x_{1}, \ldots, x_{n-1}, \ldots$ is the orbit of a point $x_{0}$. Setting $\delta_{i}=$ $\partial f_{a}^{i}\left(x_{0}\right) / \partial a$ we have $\delta_{i}=1-2 \delta_{i} x_{i}$, with $\delta_{0}=0, \delta_{1}=1$. If $a$ is a boundary point of the set of $a$ such that $f_{a}$ has an orbit of period $n, f_{a}$ is undergoing a "fold bifurcation" (see $[\mathbf{1}, \mathbf{6}]$ ). Then $\nu\left(f_{a}\right)=\beta(n)$ where

$$
\beta(n)=\left\{1-t-t^{2}+(1-t)\left(t^{3}+t^{5}+\cdots+t^{n-2}\right)\right\} /\left(1-t^{n}\right),
$$

and close to 0 we have a periodic point $x_{0}>0$ of period $n$ such that under iteration of $f_{a}^{n}$ the interval $\left[0, x_{0}\right]$ converges to $x_{0}$.

Following Milnor and Thurston we define the kneading sequence of $x$ as follows. Let

$$
\epsilon_{i}(x)= \begin{cases}+1 & \text { if } f_{a}^{\prime}\left(f_{a}^{i}\left(x_{0}\right)\right)>0, \\ -1 & \text { if } f_{a}^{\prime}\left(f_{a}^{i}\left(x_{0}\right)\right)<0 \\ 0 & \text { if } f_{a}^{\prime}\left(f_{a}^{i}\left(x_{0}\right)\right)=0\end{cases}
$$

and let $\theta_{i}(x)=\epsilon_{0}(x) \epsilon_{1}(x) \ldots \epsilon_{i}(x)$. Finally let

$$
\theta(x)=\sum_{i=0}^{\infty} \theta_{i}(x) t^{i}
$$

Then $\theta: I_{a} \rightarrow \mathbf{Z}[[t]]$ is a monotone decreasing function when we take lexicographic ordering on $\mathbf{Z}[[t]]$. In fact the coefficients of $\theta(x)$ are all \pm 1 or 0 , and if a zero occurs, all subsequent coefficients are zero. Furthermore, let $\tau: \mathbf{Z}[[t]] \rightarrow \mathbf{Z}[[t]]$ be the shift transformation

$$
\tau \sum_{i=0}^{\infty} \theta_{i} t^{i}=\sum_{i=1}^{\infty} \theta_{0} \theta_{i} t^{i-1} .
$$

Then if $x \neq 0$ we have $\theta\left(f_{a}(x)\right)=\tau \theta(x)$. This and the fact that $\theta\left(x_{0}\right)= \pm \beta(n)$ (see [5]) allows us to determine the relative positions of the points $x_{0}, x_{1}, \ldots, x_{n-1}$ in the orbit of $x_{0}$.

First of all, $\tau^{n}(\beta(n))=-\beta(n)$ whence $\theta\left(x_{0}\right)=-\beta(n)$. Straightforward calculation then reveals that the kneading coordinates of $x_{0}, \ldots, x_{n-1}$ are in the following lexicographic order

$$
\begin{aligned}
\theta\left(x_{1}\right) & <\theta\left(x_{n-1}\right)<\theta\left(x_{n-3}\right)<\cdots<\theta\left(x_{6}\right)<\theta\left(x_{4}\right)<-\theta\left(x_{2}\right) \\
& <\theta\left(x_{3}\right)<\theta\left(x_{5}\right)<\cdots<\theta\left(x_{n-4}\right)<\theta\left(x_{n-2}\right)<\theta\left(x_{0}\right)<0 .
\end{aligned}
$$

Since $\theta\left(-x_{2}\right)=-\theta\left(x_{2}\right)$ this implies that

$$
0<x_{0}<x_{n-2}<\cdots<x_{5}<x_{3}<-x_{2}<x_{4}<x_{6}<\cdots<x_{n-1}<x_{1} .
$$

Now $\left|f_{a}(y)-f_{a}(x)\right|=\left|a-y^{2}-a+x^{2}\right|=|y-x||y+x|$. In other words, if $f_{a}$ is monotone on the interval $[x, y]$ then its image is longer than $[x, y]$ by the factor 
$|y+x|$. We apply this idea to two calculations.

$$
\frac{x_{1}-x_{2}}{x_{1}-x_{0}}=x_{1}+x_{0}
$$

But $x_{1}-x_{2}>x_{1}+x_{0}$, and so $x_{1}-x_{0}>1$. Thus $x_{1}>1$. Also,

$$
\frac{x_{4}-x_{3}}{-x_{2}-x_{3}}=x_{3}-x_{2} \text {. }
$$

But $x_{4}-x_{3}>-x_{2}-x_{3}$, and so $x_{3}-x_{2}>1$. Since $-x_{2}>x_{3}$ this implies $-x_{2}>\frac{1}{2}$.

We now calculate $\delta_{n}$.

$$
\delta_{2}=1-2 x_{1} \delta_{1}=1-2 x_{1}<-1 ; \quad \delta_{3}=1-2 x_{2} \delta_{2}<0 .
$$

We continue by induction: If $\delta_{k}<0$ for odd $k<n$, then because $x_{k}>0$ we have $\delta_{k+1}=1-2 x_{k} \cdot \delta_{k}>1$. But then because $x_{k+1}>-x_{2}>\frac{1}{2}$, we have $\delta_{k+2}=1-2 x_{k+1} \cdot \delta_{k+1}<0$. Thus,

$$
\frac{\partial}{\partial a} f_{a}^{n}\left(x_{0}\right)=\delta_{n}<0
$$

Because $f_{a}$ is undergoing a fold bifurcation at $x_{0}$ and because $x_{0}>0$, the graph of $f_{a}^{n}$ has the following form on a neighbourhood of $\left[0, x_{0}\right]$ (as opposed to the graph obtained by rotating $180^{\circ}$ ).

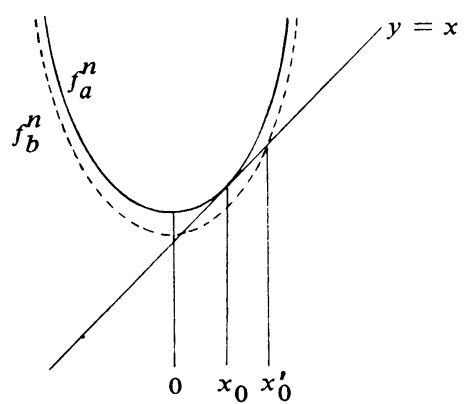

Since $\partial f_{a}^{n}\left(x_{0}\right) / \partial a<0$, we see that for $b>a$ with $|b-a|$ sufficiently small the graph of $f_{b}^{n}$ meets $y=x$ in a point $x_{0}^{\prime}$ of period $n$. This proves the theorem.

ACKNOWLEDGEMENT. This work was supported by a grant from the National Science and Engineering Council of Canada (NSERC).

\section{REFERENCES}

1. J. Guckenheimer, Bifurcations of maps of the interval, Invent. Math. 39 (1977), 165-178.

2. - Sensitive dependence on initial conditions for one-dimensional maps, Comm. Math. Phys. 70 (1979), 133-160.

3. L. Jonker, Periodic orbits and kneading invariants, Proc. London Math. Soc. 39 (1979), 428-450.

4. L. Jonker and D. Rand, The periodic orbits and entropy of certain maps of the unit interval, J. London Math. Soc. (2) 22 (1980), 175-181.

5. __ Bifurcations in one dimension; I, The nonwandering set, Invent. Math. 62 (1981), 347-365.

6. ___ Bifurcations in one dimension; II, A versal model for bifurcations, Invent. Math. 63 (1981), $1-15$.

7. J. Milnor and W. Thurston, On iterated maps of the interval, Princeton University and the Institute for Advanced Study, Princeton, N.J., 1977, preprint.

DePARTMENT OF MATHEMATICS AND STATistics, QUEEN'S UNIVERSity, Kingston, ONTARIO, K7L 3N6, CANADA 